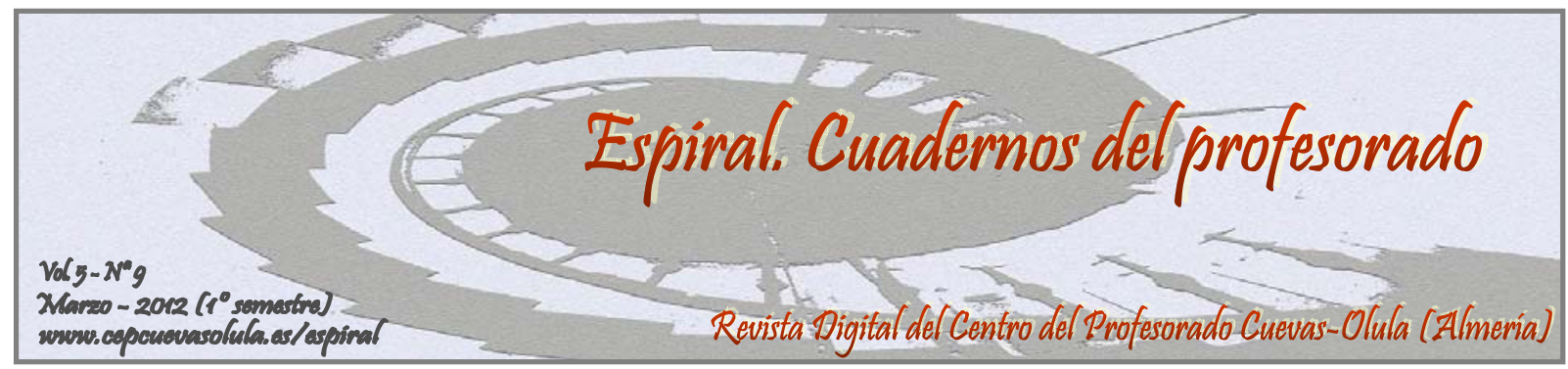

\title{
EL APRENDIZAJE COLABORATIVO COMO HERRAMIENTA PARA LOGRAR EN LA UNIVERSIDAD EL ACERCAMIENTO AL MUNDO PROFESIONAL
}

\section{COLLABORATIVE LEARNING AS A TOOL FOR SUCCESSFUL PROFESSIONAL ORIENTATION IN THE UNIVERSITY}

\author{
Verónica Baena Graciá $^{(1)}$, Jaime Angulo Zevallos ${ }^{(1)}$, Daniel Gualoto ${ }^{(1)}$, \\ Vicente Padilla Valencia ${ }^{(1)}$ y Javier Sanz Blasco ${ }^{(1)}$
}

\author{
${ }^{(1)}$ Universidad Europea de Madrid, España
}

RESUMEN: La Formula Student es el mayor evento europeo del mundo del motor con perfil docente. Desde el año 2007, la Universidad en la que trabajan los autores participa en esta competición mediante la elaboración de un monoplaza que participó en la competición Formula Student: el UEM01. El proyecto siguió desarrollándose el año siguiente con la fabricación del UEM02 que alcanzó una meritoria posición en el circuito de Montmeló. En 2011, -para la fabricación del UEM03-, junto a los estudiantes de ingeniería estudiantes y profesores de otras Facultades (Artes y Comunicación y Ciencias Sociales) se han unido al proyecto. A través de esta actividad, los alumnos desarrollaron diversas tareas (diseño del coche, fabricación del monoplaza, búsqueda de patrocinadores, elaboración de campañas de difusión en los medios, etc.) siendo lo más parecido posible al mundo real. El objetivo reside, por tanto, en la elaboración de una actividad que permita a los alumnos desarrollar competencias esenciales en el mundo laboral y acercarles al mundo profesional. Los excelentes resultados obtenidos confirman la idoneidad de esta actividad ya que no sólo logró los objetivos previstos sino que además, incrementó la motivación del alumnado, su rendimiento académico y nivel de implicación en el aula.

Palabras clave: Innovación Docente; Aprendizaje Cooperativo; Trabajo en Equipo; Metodologías Activas de Aprendizaje; Espacio Europeo de Educación Superior.

ABSTRACT: The Formula Student is the largest European event in the world of teaching profile engine. Since 2007, University where the authors of this research belong to, was involved in this competition with the aim of encouraging students to implement all the knowledge acquired in the classroom. The UEM01 was then developed to participate in the Formula Student competition. The project continued to develop the following year with the manufacture of UEM02, which reached a good position in the Montmeló circuit. In 2011 - for the manufacture of UEM03 - apart from the engineering students both students and teachers from other Schools (Arts and Communication, and Social Sciences) have joined the project. Through this activity, students have developed various tasks (car design, car manufacturing, finding sponsors, developing campaigns in the media, etc.) being as close as possible to the real world. Therefore, the purpose of this study is to develop an activity that allows students to develop essential skills in the workplace and bring them into the professional world. The obtained findings confirm the suitability of this activity not only to achieve its objectives but also to increase the students' motivation, academic performance and level of involvement in the classroom.

Key words: Teaching Innovation; Cooperative Learning, Teamwork, Active Learning Methodologies; European Higher Education Area 
Baena Graciá, V., Angulo Cevallos, J., Gualoto, D., Padilla Valencia, V., y Sanz Blasco, J. (2012). El aprendizaje colaborativo como herramienta para lograr en la universidad el acercamiento al mundo profesional. Espiral. Cuadernos del Profesorado, 5(9), 47-54. Disponible en: http://www.cepcuevasolula.es/espiral.

Fecha de recepción: 25/10/2011

Fecha de aceptación: 13/12/2011
Enviar correspondencia a: veronica.baena@uem.es

\section{1.- INTRODUCCIÓN}

De acuerdo con los planteamientos que inspiran el proceso de Convergencia Europea de la Educación Superior, los escenarios y las metodologías de la enseñanza universitaria deben experimentar una profunda renovación (De Miguel, 2009). La principal está relacionada con las titulaciones, las cuales deben ser diseñadas de un modo que permita armonizar los títulos en toda Europa y den respuesta a las necesidades actuales y futuras tanto de los estudiantes como del mercado de trabajo. Sin embargo, otro de los cambios introducidos por el Espacio Europeo de Educación Superior (EEES) es la apuesta por focalizar la atención en la formación de los estudiantes y concretamente, en su desarrollo competencial (Cano, 2008).

Según Medina (2009, p. 10-11), "las competencias son los logros evidentes que adquieren los seres humanos al conocer, actuar y resolver los problemas en las más diversas situaciones personales, institucionales y profesionales”. Su estudio debe estar presente en los programas formativos del siglo XXI y provocar un cambio en el sistema de aprendizaje global universitario. En otras palabras, frente a los enfoques didácticos clásicos centrados en el aula y en la actividad profesional del profesor, hoy se propugna una enseñanza basada en el estudiante. Además, la existencia de un mercado laboral cada vez más competitivo, hace necesario que las Universidades profundicen en conocer a su alumnado para poder adaptar sus programas y metodologías docentes en los nuevos títulos de grado y posgrado (Barber, 2008; Pusztai y Szabó, 2008). Por ello, es necesario cambiar la tradicional dinámica de enseñanza basada en el aprendizaje memorístico de conocimientos (Zabala y Arnau, 2008) por otra donde los estudiantes sean una parte activa, asuman responsabilidades y pasen de sujetos pasivos a protagonistas de su propio aprendizaje (Whitehead, 2008), quedando así preparados para los cambios que se producen en el entorno social y profesional.

En otras palabras, la Universidad debe tener en cuenta que sus estudiantes no sólo necesitan conocer en profundidad los contenidos de las asignaturas que cursan sino además, ser capaces de tener un conocimiento integrado, interdisciplinar e incluso, intercultural que puedan aplicar y poner en práctica para resolver problemas reales (Medina, 2009). Sólo así, serán capaces de desenvolverse en un entorno de trabajo cambiante, competitivo y complejo (Hunt, Eagle y Kitchen, 2004, entre otros). Por ello, los profesores universitarios no deben limitarse a la mera transmisión de conocimiento a su alumnado sino que por el contrario, han de aplicar su programa formativo al mundo real, contribuir al desarrollo competencial de sus alumnos y asegurarse de que sean capaces de aplicar dichas competencias en el mundo laboral (Canzer, 1997; Baena, 2010).

No son muchas las actividades universitarias realizadas para incrementar la conexión con el mundo real sin perder de vista el desarrollo competencial del alumnado y el fomento de la internacionalidad. Este trabajo pretende cubrir este vacío y por ello, describe una actividad docente llevada a cabo entre 5 profesores y 74 alumnos de la Escuela Politécnica, la Facultad de Artes y Comunicación y la Facultad de Ciencias Sociales de la Universidad donde trabajan los autores durante el curso 2010/2011: la construcción de un coche de Formula 1 por parte de los alumnos de ingeniería, el diseño del su carrocería por los alumnos de comunicación y la puesta en práctica de una estrategia 
de marketing y patrocino a cargo de los alumnos de marketing. Todo ello, bajo el marco de la Formula SAE, en general, y la Formula Student, en particular.

La Formula SAE, es el mayor evento del mundo del motor con perfil educativo. Concretamente, se trata de una competición entre alumnos de universidades de todo el mundo que promueve la excelencia en ingeniería a través de una competición entre universidades que diseñan, construyen, desarrollan y compiten como un pequeño equipo con un monoplaza. La primera competición se produjo en 1979 cuando Mark Marshek, profesor de la Universidad de Houston, contactó con el Departamento de Relaciones Educativas de la SAE un año antes. El proyecto fue un éxito y en 1981, se organizó en la Universidad de Texas la primera edición de la Formula SAE. Participaron 6 equipos y un total de 40 alumnos. Esta competición ha ido creciendo y desde 1998 también se celebra en Warwickshire una edición británica conocida como Formula Student. Actualmente, se celebran competiciones en numerosos países como Alemania, Austria, España, Japón, Brasil, Australia, etc. que llegan a albergar hasta 120 equipos y más de 2.000 estudiantes, los cuales deben fabricar un monoplaza a la vez que realizar un plan de producción, marketing y costes para la fabricación del mismo y de una serie de 1.000 unidades anuales. Para ello, junto a los conocimientos técnicos, los estudiantes desarrollan destrezas en otras áreas tales como gestión, comercialización, gestión de costes, diseño, etc. Ámbitos vitales en todos los sectores de empleo.

Así pues, en la Formula Student participan estudiantes de las mejores universidades europeas, cuyo reto es la aplicación de conocimientos técnicos y trabajo en equipo con el objetivo diseñar y construir un monoplaza de competición, que se enfrentará en pruebas estáticas y dinámicas a los diferentes equipos. Además de este tipo de pruebas, también es puntuable para ganar la competición la viabilidad económica del proyecto, y la presentación que cada equipo hace de su prototipo. Una de las máximas de este proyecto, -el cual está apoyado por empresas punteras en tecnología, que apuestan por el desarrollo educacional y profesional como por ejemplo, Airbus, Mercedes-Benz, Shell o EOn-, es que los alumnos tengan una actividad lo más parecida posible a la que desarrollarán una vez se incorporen al mundo laboral. Para ello el equipo tiene una estructura muy organizada tanto con equipos divididos según distintas especialidades (Chasis, Electrónica, Suspensión, etc.) como con una jerarquía que imita el método de los grupos de trabajo de cualquier empresa.

$$
\text { La presencia de }
$$
equipos españoles en la Formula Student es todavía

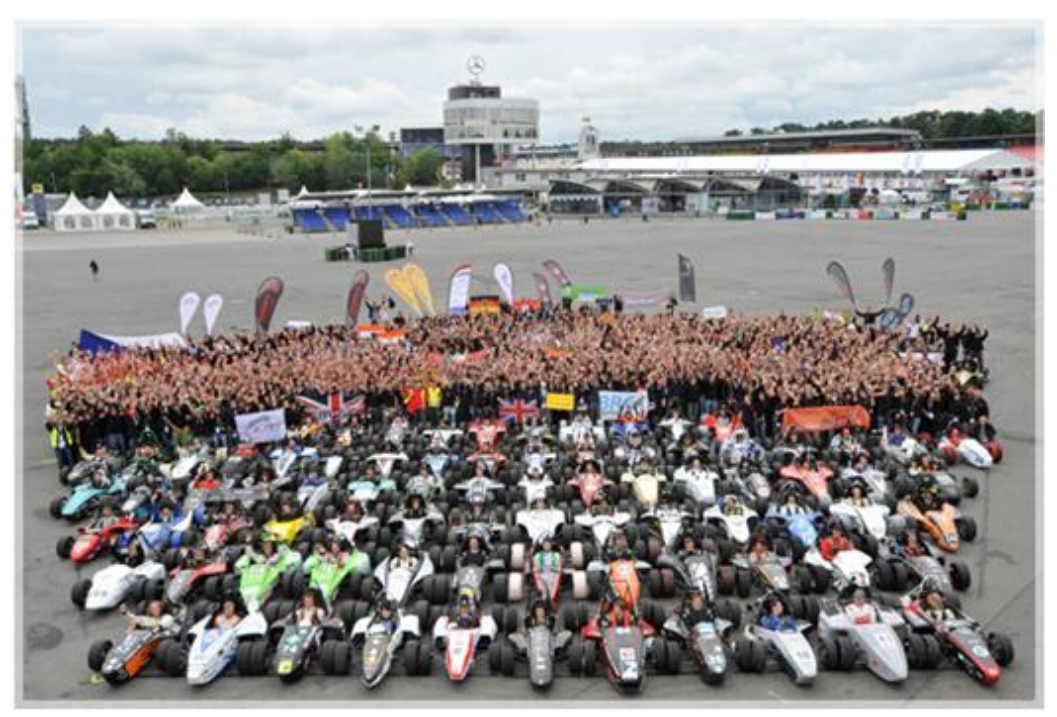

Figura 1.- Equipos participantes en la Formula Student discreta en comparación con la de las grandes potencias como Alemania, Japón y Estados Unidos. Por ejemplo, en el evento organizado en septiembre del 2010, la victoria fue para el equipo alemán Horsepower Hannover de la Universidad de Hanover. El primer equipo español fue ETSEIB Motorsport, de la Universidad Politécnica de Cataluña, quedando en quinta posición. No obstante, cada vez es mayor el número de universidades españolas que se han ido incorporando al proyecto, como la Universidad de Navarra, la Universidad del País Vasco y la Universidad Politécnica de Madrid, por lo que cabe esperar mejores resultados de nuestros equipos en un futuro no muy lejano. 
Desde el año 2007, la Universidad a la que pertenecen los autores participa en esta competición a través del equipo FomulaUEM, con el objetivo de impulsar a los alumnos a la puesta en práctica de todos los conocimientos adquiridos en las clases. El resultado fue la elaboración de un monoplaza que participó en la competición Formula Student, el UEM01. El proyecto siguió desarrollándose el año siguiente con la fabricación del UEM02, que alcanzó una meritoria posición en el circuito de Montmeló. Durante el curso académico 2010/2011, el equipo de FormulaUEM estuvo compuesto por 5 profesores y 74 alumnos de la Escuela Politécnica, la Facultad de Artes y Comunicación y la Facultad de Ciencias Sociales, cuyo objetivo es la elaboración del UEM03. Los 74 alumnos están distribuidos en 7 grupos de trabajo comandados por un "team leader”. Además, cada equipo tiene un "manager", responsable de las funciones del mismo.

De este modo, el objetivo de este trabajo reside en la presentación y análisis de resultados de una actividad de innovación docente de carácter multidisciplinar llevada a cabo en una Universidad española durante el curso 2010/2011, que permitió a los alumnos desarrollar competencias esenciales en el mundo laboral (tales como el trabajo en equipo, la gestión de conflictos o la iniciativa, entre otras) y acercarles al mundo profesional de forma amena.

\section{2.- METODOLOGÍA}

Para lograr los objetivos propuestos en la sección anterior, el equipo de FormulaUEM está compuesto por profesores que coordinan y dirigen el proyecto gracias a su amplia y dilatada trayectoria experiencia académica y laboral (Academy Staff). La coordinación general y representación de la Universidad en las competiciones está encomendada a la figura del "Faculty Advisor" cuya presencia en las competiciones oficiales es obligatoria ya que se responsabiliza tanto de la veracidad de los documentos técnicos como de la seguridad y orden del equipo en cada evento. Además de esta figura obligatoria en la competición, los cinco profesores implicados en la FormulaUEM tienen las siguientes funciones:

1. Financial \& Marketing Advisor: Asesora y dirige a los alumnos en todo lo referente a la creación de marca, visibilidad del equipo en los medios de comunicación y formulación de estrategias para atraer patrocinadores interesados en invertir en la FormulaUEM.

2. Acting Powertrain \&Performances Advisor: Asesora y dirige al equipo en todo lo relativo a motor y trasmisión. También define la estrategia para cada evento dinámico.

3. Mody\&Design Advisor: Asesora y dirige al grupo de alumnos en cuanto al diseño y construcción de carrocería así como todo lo referente a estética del monoplaza.

4. Vehicle Advisor: Proporciona asesoramiento en todo lo referente a geometría del vehículo.

5. Manufactory Advisor: Asesora a los alumnos en todo lo concerniente a fabricación y ensamblaje del monoplaza.

Para lograr los objetivos planteados en el equipo (construcción de un monoplaza), los alumnos no sólo participaron en un ambicioso programa de mejoras en la construcción del UEM03 (para lo cual se han impartido hasta 960 horas de formación en cursos gratuitos de manejo de máquinas, herramientas, motores, etc.) sino además, formaron grupos de trabajo multidisciplinares responsables de la ejecución de diversas funciones como el diseño de la carrocería, la difusión del FormulaUEM en los medios de comunicación o la captación de patrocinadores, entre otras acciones. Todo ello, - bajo la tutela y control de los profesores implicados en este proyecto -, dotó a los estudiantes de la experiencia y el conocimiento necesario para acceder en condiciones óptimas al mercado de trabajo.

Además, esta actividad se diseñó con el objetivo de reforzar los elementos esenciales del modelo académico promovido en el EEES. Para lograrlo, se pusieron en práctica las siguientes acciones:

- Situar al alumno en el centro del proceso formativo. En concreto, la FormulaUEM has sido diseñada y llevada a cabo para fomentar la adquisición y desarrollo de competencias por parte del alumnado a través de su participación en diversas tareas que requieren trabajo en equipo, integración 
de la información, espíritu crítico, capacidad de análisis, liderazgo, iniciativa, creatividad y habilidades comunicativas, entre otros aspectos.

- Fomento de la internacionalidad ya que los alumnos compiten con las mejores universidad del mundo, creándose un agradable ambiente de intercambio de experiencias e incluso, colaboración. Además, dado que todas las presentaciones se realizan en Inglés, los alumnos impulsan el desarrollo de esta lengua.

- La FormulaUEM es un proyecto atractivo que acerca el mundo real a sus alumnos, lo que redunda positivamente en la adquisición de conocimientos por parte de los estudiantes implicados en el equipo, incrementa su rendimiento académico y eleva la motivación en el aula.

- Por otra parte, para que el equipo funcione correctamente, los alumnos cuentan en todo momento con el apoyo del Academy Staff, profesores que coordinan y dirigen el proyecto gracias a su amplia y dilatada trayectoria experiencia académica y laboral. El objeti-

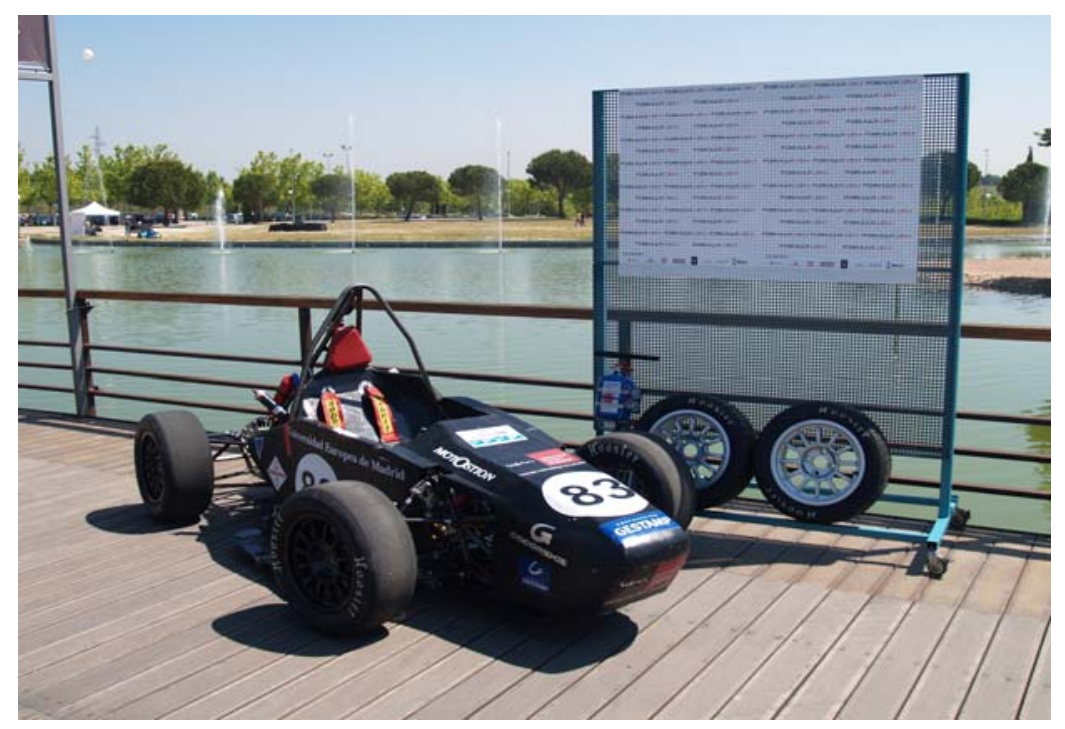

Figura 2.- Presentación oficial de UEM03 en el Campus Universitario vo de esta colaboración reside en la elaboración de un nuevo monoplaza - denominado UEM03 - que contase con grandes prestaciones técnicas y el mejor diseño. En concreto, los alumnos del Grado en Ingeniería Mecánica, Ingeniería Industrial y doble titulación Ingeniería Industrial + ADE elaboraron un monoplaza siguiendo las indicaciones de los alumnos del Grado en Diseño (Mención Diseño de Interiores), titulación perteneciente a la Facultad de Artes y Comunicación. Esta colaboración no sólo permitió a los alumnos de ambas titulaciones poner en práctica los conocimientos teóricos adquiridos en un proyecto real sino que además, les dio la oportunidad de llevar a cabo las siguientes actividades:

- Aprender a integrarse en un equipo multidisciplinar y global, intercambiando conocimientos, inquietudes y respetando otras prioridades.

- Conocer cómo se estructura y proyecto de gran recorrido y en equipo, por fases, ajustado a un cronograma de trabajo del que se responsabilizan.

- Realizar una tarea investigación orientada a restricciones, referencias y tendencias, que les permitió plantear una estrategia de posicionamiento de producto innovadora.

- Elevar su conocimiento en el uso de herramientas informáticas, debido a la complejidad del proyecto.

- Enfrentarse a problemas, de experimentar y de fabricar ellos mismos con distintos métodos y sistemas productivos.

- Adelantarse a los problemas y a tomar nota de los errores propios y ajenos. Mejorando en autoestima y confianza.

- Experimentar su constancia y capacidad de trabajo intensiva, sometidos al rigor de un calendario de entrega y los niveles de calidad consensuados.

- Ser testigos de la interacción que se produce entre gestores, proveedores y patrocinadores a la hora de llevar un trabajo a buen puerto.

- Trabajar conceptos de fotografía, escenografía y comunicación muy cercanos al uso profesional de primer nivel. 
Fruto de este trabajo, se produjo la presentación del UEM03, como se recoge en la Figura 2.

Junto a lo anteriormente expuesto, el grupo profesores y alumnos de la Escuela Politécnica participantes en el equipo de FormulaUEM no sólo contó con la ayuda de la Facultad de Artes y Comunicación (labores de diseño y construcción de carrocería) sino además, con la asistencia de la Facultad de Ciencias Sociales en la estrategia de marketing del equipo. En concreto, los alumnos de la doble titulación de Administración y Dirección de Empresas (ADE) + Investigación y Técnicas de Mercado (ITM) colaboraron en la elaboración de una campaña real de marketing para lograr la mayor difusión y apoyo de patrocinadores al proyecto de FormulaUEM. Para lograr la máxima notoriedad y conseguir el apoyo de empresas que patrocinasen el proyecto, los alumnos se asociaron en diferentes equipos de trabajo y realizaron las siguientes tareas:

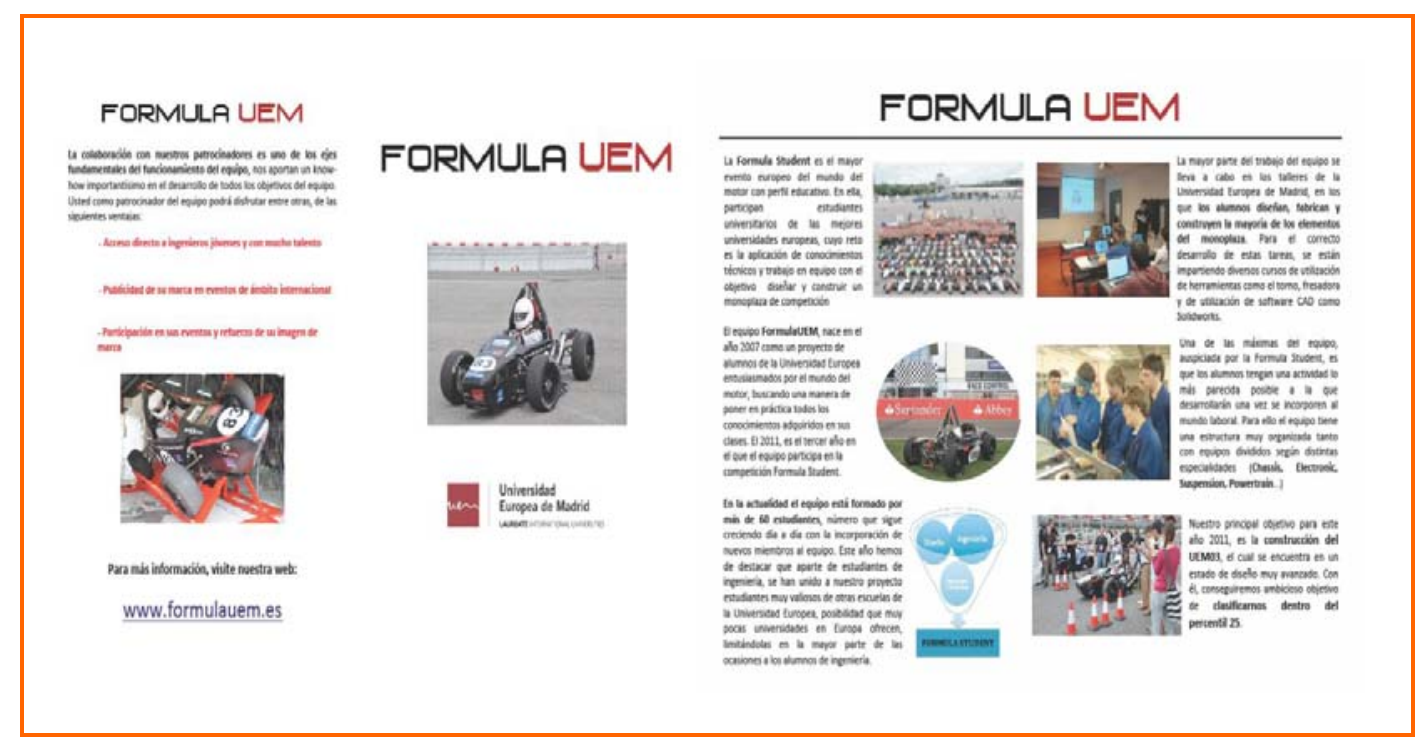

Figura 3.- Ejemplos del dossier y folleto informativo elaborados

- Elaboración de dossier informativo y folletos explicativos del proyecto que animase a las empresas a convertirse en patrocinadores del equipo de FormulaUEM.

- Asesoramiento sobre el diseño y gestión de la página web del equipo:

http://www.formulauem.es a través de la cual se informa de las novedades, se cuelgan videos del coche grabados por los alumnos, se muestra el organigrama del equipo, así como el listado de entidades patrocinadoras del equipo, entre otros aspectos.

- Creación de eventos que permitiesen dar difusión del proyecto y recaudar fondos para sufragar los gastos de elaboración del monoplaza, asistencia a las competiciones, etc. Para ello, se organizaron diferentes eventos. Algunos de ellos lograron gran cobertura en los medios de comunicación. Tal es el caso del día de la presentación del UEM03, el cual recibió el nombre de "El Día de la FormulaUEM" y estuvo acompañado de diferentes actividades como sorteos, concierto de un grupo de música, pruebas con el kart o competiciones entre coches deportivos y el monoplaza en el parking de la Universidad. Fruto de esta jornada, el equipo de FormulaUEM logró una importante difusión diferentes medios de comunicación como la televisión (MarcaTV), la radio (cadena COPE), la prensa física (diario AS) y diferentes medios de prensa digital. Para anunciar este evento, los alumnos de la Facultad de Ciencias Sociales grabaron un video en con la ayuda técnica de los alumnos de la Facultad de Artes y Comunicación que en sólo dos días recibió más de 200 visitas. Además, es preciso señalar que el evento estuvo patrocinado por el grupo Sodexo que ofreció un catering para 200 personas. También se contó con la colaboración de la bebida Burn (The Coca Cola Company) y se repartieron botellas entre los asistentes a las diferentes actividades de "El Día de la FormulaUEM". 
- Informar a través de redes sociales (facebook, twitter y tuenti) de todas las novedades del equipo de FormulaUEM.

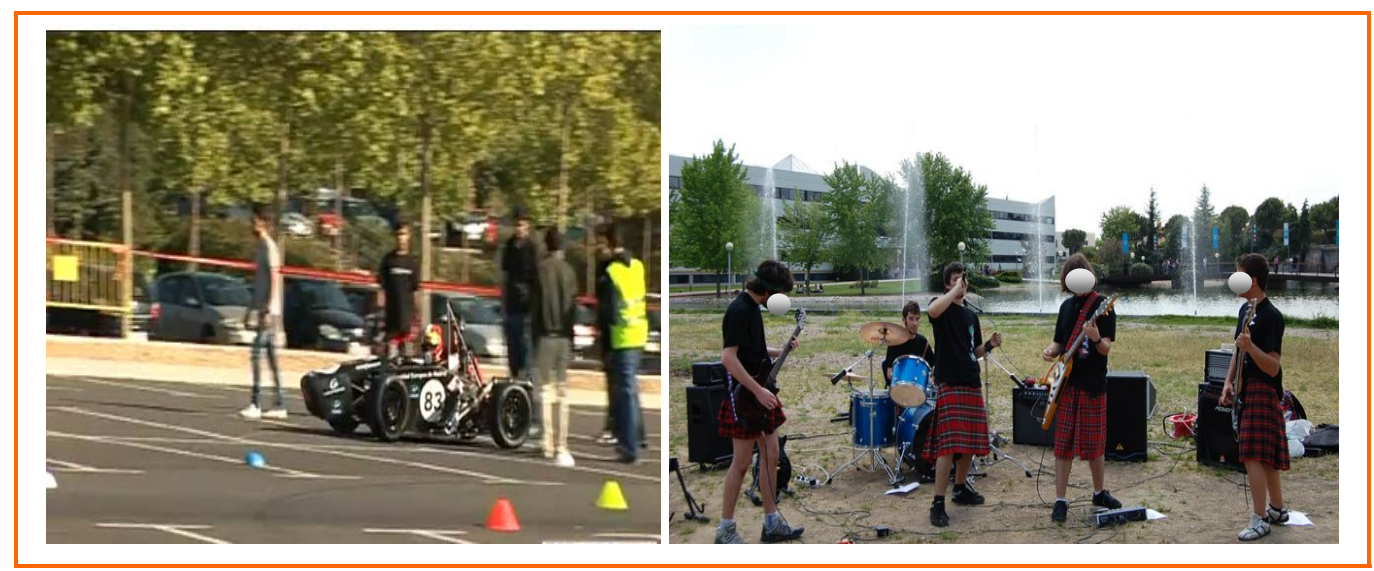

Figura 4.- Diferentes escenas el "El Día de la FormulaUEM”

\section{3.- RESULTADOS}

Los profesores participantes en el equipo de la FormulaUEM pudieron comprobar los excelentes resultados obtenidos. En concreto, los alumnos se mostraron implicados desde el principio y mostraron una actitud proactiva. Por ello, nos atrevemos a afirmar que la FormulaUEM coadyuvó al aprendizaje activo por parte de los estudiantes e incrementó su motivación en el aula y rendimiento académico.

Además, durante el desarrollo de la actividad los alumnos tuvieron la oportunidad de poner en práctica los conocimientos teóricos aprendidos en cursos anteriores. Asimismo, todos los estudiantes implicados en el proyecto mostraron una actitud colaborativa con sus compañeros de trabajo. Pero esas no fueron las únicas competencias que desarrollaron, ya que el hecho de tener que trabajar en equipos multidisciplinares (compuestos por alumnos de diversas titulaciones y Facultades) hizo que pudiesen reforzar sus habilidades comunicativas, integración de la información, responsabilidad, organización, planificación, creatividad, iniciativa, gestión de conflictos y por supuesto, el trabajo en equipo.

Por otra parte, la FormulaUEM permitió que los alumnos se acercasen al mundo profesional (cada uno en su especialidad) siendo responsables de aspectos tan importantes como la fabricación de un monoplaza (chasis, suspensión, powertrain), el diseño de la carrocería e interiores y la elaboración de una campaña marketing para captar patrocinadores (englobando aspectos tan diversos como el diseño de dossier y folletos informativos, la creación y gestión de una página Web, la planificación y puesta en marcha de eventos y su comunicación a través de redes sociales).

En otras palabras, la actividad llevada a cabo no sólo facilitó la adquisición por parte de los alumnos de una visión integradora de contenidos sino que además, coadyuvó al desarrollo y refuerzo de muchas competencias requeridas en el mundo profesional. Además, permitió que los estudiantes se acercasen al mundo real, incorporasen la internacionalidad en su perfil curricular y fuesen protagonistas activos de su propio proceso de aprendizaje logrando con ello, resultados congruentes a los objetivos estratégicos establecidos en Bolonia para el EEES.

\section{4.- CONCLUSIONES}

Este trabajo describe una actividad de innovación docente llevada a cabo durante el curso académico 2010/2011 en la que participaron 5 profesores 74 estudiantes de diferentes titulaciones y tres Facultades de la Universidad a la que pertenecen los autores de este trabajo (Escuela Politécnica, Facultad de Artes y Comunicación y Facultad de Ciencias Sociales). En ella, alumnos y profesores de diferente perfil académico colaboraron en un mismo proyecto (el equipo de FormulaUEM) lo que 
incrementó las relaciones entre Escuelas/Facultades de nuestra Universidad y proporcionó un diferencial curricular en los alumnos participantes en el proyecto. Asimismo, al participar en competiciones internacionales, el proyecto de FormulaUEM favoreció la orientación internacional de los alumnos implicados en el proyecto.

Junto a ello, la participación en el equipo de FormulaUEM permitió a los estudiantes adquirir y desarrollar competencias esenciales en el mundo laboral, tales como el trabajo en equipo, la integración de la información, la creatividad, la gestión de conflictos, la iniciativa y las habilidades comunicativas, ente otras. Esto incrementó su motivación, satisfacción en el aula y rendimiento académico.

\section{5.- REFERENCIAS}

Baena, V. (2010). Innovación docente e identificación de inhibidores del aprendizaje en el área de empresa. Una propuesta metodológica, Espiral - Cuadernos del Profesorado, 3(6), 3-14- Disponible en www.cepcuevasolula.es/espiral.

Barber, M. (2008). A formula for great teaching, Times Educational Supplement, 48, 19-29.

Cano, M.E. (2008). La evaluación por competencias en la educación superior. Universitat de Barcelona. Disponible en línea: http://www.ugr.es/local/recfpro/rev123COL1.pdf. [Consulta: 4 de mayo de 2010]

Canzer, B. (1997). Marketing education on the internet: a world wide web based introductory marketing course design for the virtual project in distance education at Simon Fraser University. Journal of Marketing Education , 23, 56-65.

De Miguel, M. (2009). Metodologías de enseñanza y aprendizaje para el desarrollo de competencias. Orientaciones para el profesorado universitario ante el Espacio Europeo de Educación Superior, Madrid: Alianza Editorial.

Hunt, L., Eagle, L., y Tchen, P. (2004). Balancing marketing education y information technology: matching needs or needing a better match? Journal of Marketing Education, 26, 75-88.

Medina, A. (2009). Formación y desarrollo de las competencias básicas. Madrid: Editorial Universitas, S.A.

Pusztai, G., y Szabó, P.C. (2008). The Bologna process as a Trojan horse. European Education, 40, 85-103.

Whitehead, D.P. (2008). Thoughts on Education y Innovation. Childhood Education, 85, 106-118

Zabala, A., y Arnau. L. (2008) Cómo aprender y enseñar competencias, Barcelona: Graó. 